\title{
Class-B Microwave-Photonic Link Using Optical Frequency Modulation and Linear Frequency Discriminators
}

\author{
Thomas E. Darcie, Fellow, IEEE, Jinye Zhang, Peter F. Driessen, Senior Member, IEEE, and Jae-Jeong Eun
}

\begin{abstract}
A class-B optical link is demonstrated as a means to provide high spur-free dynamic-range $\left(110.4 \mathrm{~dB} \cdot \mathrm{Hz}^{2 / 3}\right)$ with a minimum average received power associated with the optical carrier. The link uses optical frequency modulation, linear fiberBragg-grating frequency discriminators, and balanced detection to provide high linearity and maximum signal power. Shot and intensity noises are minimized by the low total average received photocurrent $(0.88 \mathrm{~mA})$ that results from class-B operation.
\end{abstract}

Index Terms-Analog links, fiber Bragg grating (FBG), microwave-photonic links (MPLs), optical frequency discriminator, optical frequency modulation (FM), spur-free dynamic range (SFDR), subcarrier modulation.

\section{INTRODUCTION}

W HILE microwave-photonic links (MPLs) have become essential for numerous lightwave system applications such as radar systems, radio-over fiber networks, and video distribution networks, nonlinearity and noise persist as primary limitations. Research continues to push link performance metrics like spur-free dynamic range (SFDR) or carrier-to-noise ratio (CNR) [1]. A key impairment is noise associated with the optical carrier that results from prebiasing the modulated optical source at a linear operating point [2]. This carrier creates shot and intensity noises that are dominant noise sources in high-SFDR links in which the total received optical powers may approach $100 \mathrm{~mW}$. Beat noise arising between amplified spontaneous emission and this carrier limits the usefulness of optical amplifiers. High carrier powers saturate and damage photodetectors, limiting the magnitude of the much smaller modulated signal powers. Fiber nonlinearities, including nonlinear refractive index $(n 2)$ and stimulated Brillouin scattering, are activated by high carrier powers, resulting in impairment and forcing the use of mitigation techniques.

Manuscript received June 30, 2006; revised September 27, 2006. This work was supported in part by the National Sciences and Engineering Research Council (NSERC), Canadian Foundation for Innovation, British Columbia Knowledge Development Fund, and the Canadian Institute for Photonic Innovation.

T. E. Darcie, J. Zhang, and P. F. Driessen are with the University of Victoria, Victoria, BC V8W 3P6, Canada (e-mail: tdarcie@uvic.ca; jinzhang@uvic.ca; peter@ece.uvic.ca).

J. J. Eun is with the Department of Electronic Engineering, Changwon National University, Changwon 641-773, Korea and also with the Department of Electronic Engineering, University of Victoria, Victoria, BC V8W 3P6, Canada (e-mail: jjeun@changwon.ac.kr).

Color versions of one or more of the figures in this paper are available online at http://ieeexplore.ieee.org.

Digital Object Identifier 10.1109/JLT.2006.888173
Methods to reduce or eliminate this carrier have therefore been the subject of considerable effort. Low-biased operation [2] of a conventional Mach-Zehnder (MZ) modulator reduced the carrier power. Unfortunately, the modulation efficiency, and hence link gain, is also reduced, and second-order distortion becomes large. Optical filtering to reduce the optical carrier [3] reduces the carrier power directly. This alone has a similar effect as increasing the modulation index, hence, increasing distortion. A variety of coherent techniques, e.g., [4], have been attempted. While the carrier may be suppressed for transmission, implementation is complex, and the noise associated with the local oscillator becomes a problem. Dynamic bias modulation [5] can result in lower average carrier powers. The magnitude of the transmitted RF power is detected (envelope or square-law) and applied to modulate the bias current. Hence, the bias point can be minimized, and on average, lower carrierrelated noise results. However, nonlinearity of the source or modulator transfer function is exacerbated by the interaction between the modulating signal and bias modulation. From these attempts, it becomes clear that the ability to reduce the carrierrelated impairment with a single link and detector is limited.

Common-mode intensity noise (that is present at the input to a modulator) can be suppressed, e.g., [6], using a dualoutput MZ, two transmission fibers, and balanced differential detection. However, the degree of intensity noise suppression is limited as the modulation index increases [7], the shot noise (uncorrelated in the two detectors) is not reduced, and the intensity noise added after the modulator (e.g., signal-spontaneous beat noise from an optical amplifier) is not suppressed. It was shown [8] that balanced low-biased operation could reduce the second-order distortion associated with low-biased operation. More recently, this approach was generalized to class- $\mathrm{AB}$ modulation [9] and shown to result in a reasonable improvement in dynamic range. Each of two MZs was operated at a highly nonlinear bias point, providing a 5.7-dB reduction of shot noise, relative to an $\mathrm{MZ}$ at the normal quadrature bias (Q-bias) point. The term "class AB" is used in an identical context to electronic amplifiers in which two complementary transistors are used. Class AB is similar to class B but with a slight dc bias. It was then shown how the class-AB system could be implemented using one transmission fiber and one polarization modulator [10]. While class $\mathrm{AB}$ provides a reasonable reduction in carrier while retaining good linearity, it was shown [11] how class-B techniques could, theoretically, result in the ultimate performance in an optical link. 


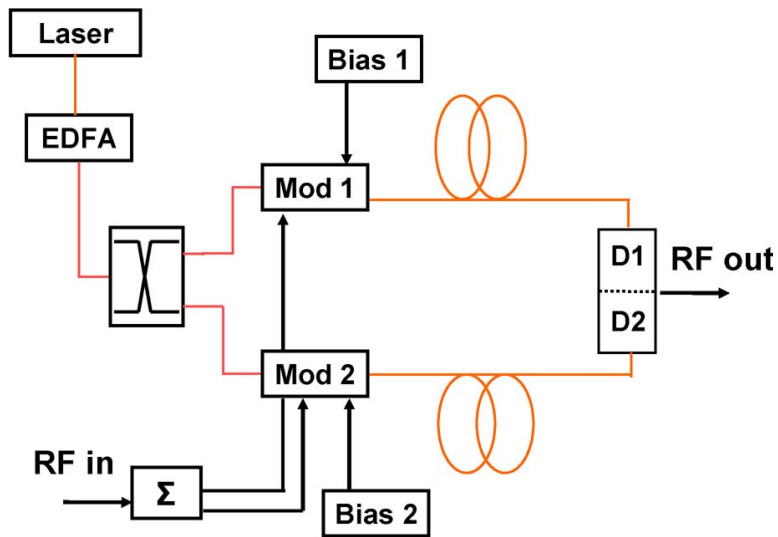

Fig. 1. Example of a class-B optical link with two complementary modulators Mod 1 and Mod 2 and differential detection in a balanced detector (D1 and D2).

In an ideal class-B optical link [11], all received optical power contributes to signal power. If suitable transfer functions for the source modulator can be realized, shot noise can be reduced by $11 \mathrm{~dB}$ and intensity noise by $20 \mathrm{~dB}$, relative to a conventional link and for a modulation index of $10 \%$. However, as described later, this requires a perfectly linear transfer function with a sharp turn-on threshold for each of the complementary source modulators. The sinusoidal voltagetransmission transfer function for a standard MZ modulator is far from ideal and is therefore limited to a class- $\mathrm{AB}$ operation. The lack of intensity modulators with suitable transfer functions has frustrated our attempts to consider class-B links in the optical intensity domain.

In this paper, we present the first experimental demonstration of what approximates a class-B MPL. We create nearly ideal class-B transfer functions for the complementary modulated sources using optical frequency modulation (FM) and customdesigned fiber Bragg gratings (FBGs). Measurements show that a high SFDR can be achieved with a small fraction of the received optical power required normally, validating the merit of a class-B operation. We describe the class-B approach in Section II and the interaction between the optical angle (phase or frequency) modulated source and frequency discriminators in Section III. The experimental setup and results are described in Sections IV and V, respectively.

\section{BACKGROUND}

In an ideal class-B system [11], as shown conceptually in Fig. 1, a zero-mean input RF signal is half-wave rectified. Positive voltage is converted linearly to intensity and transmitted on one optical link. Negative voltage is transmitted as intensity over a second matched link. A balanced optical detector is used to subtract the two detected complementary halfwave-rectified signals, resulting in the recovery of the original microwave signal, but after transmission with effectively zero dc bias.

The challenge is to create the appropriate transfer functions required to accomplish the half-wave rectification and linear modulation. Given that no suitable intensity modulator exists, we use optical FM and FBGs to provide the effective transfer function shown in Fig. 2. In simple terms, the input voltage

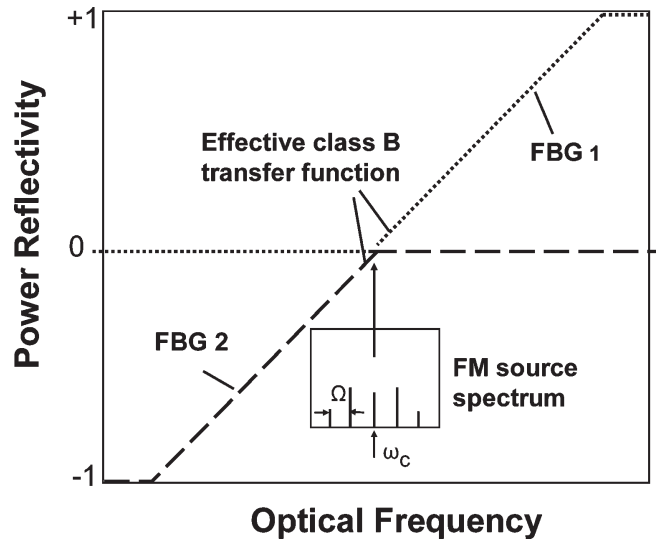

Fig. 2. Effective system transfer function when the intensity reflected from FBG2 is subtracted from that from FBG1 in balanced detector. Insert shows the frequency-modulated source spectrum for a single modulating tone at the frequency $\Omega$.

is converted linearly to FM, and this FM is converted by each FBG to intensity modulation (IM). Since the balanced detector subtracts currents generated in each detector, the reflectivity $(R)$ for FBG2 is inverted, showing that the effective ideal transfer function is linear between \pm 1 , with zero dc. This simple description captures the general principle of operation and, as we discuss later, is valid for large FM modulation indexes. Subtleties associated with this form of nonlinear FM detection somewhat complicate the situation.

By comparing a Q-biased link with an ideal class-B link, the predicted reduction [11] in mean-square shot noise power for the same signal power is given by $\sqrt{2 / \pi} \mu$, where $\mu$ is the rootmean-square (rms) modulation index. Intensity noise is reduced by $\mu^{2}$. This analysis assumes that only the half-wave-rectified signals contribute dc optical power to each detector. For class B, $\mu$ is defined by the ratio of the rms detected signal current in one detector to the maximum current (1.0 on Fig. 1). For large FM modulation index, this is equivalent to the ratio of the rms frequency deviation of the FM source to the width of the linear frequency ramp of the FBG. For a small $\mu$ typically encountered in high-SFDR links, noise reduction can be substantial. For signals that do not resemble the Gaussian amplitude probability density function used in [11], like the two-tone measurements described later in this paper, the reduction in noise can be approximated using $\mu=m \sqrt{N / 2}$, where $m$ is the modulation index (ratio of peak amplitude to dc) for each of the $N$ channels or tones ( $\mu=m$ for two tones).

The requirement for two optical paths is a practical issue that must be managed for all high-SFDR applications, where the high detected optical power leads to high levels of intensity noise. Previously demonstrated methods for suppressing intensity noise using balanced detection [6] also use two links: one for each of the two outputs of a dual-output MZ. The two optical paths must be matched to a small fraction of the RF wavelength. With class B, the two paths are required only to convey the complementary signals to the balanced detector. Intensity noise is reduced through the reduction of the optical carriers in each complementary signal, rather than through common-mode subtraction, so it is no longer required that the signals subtracted by the balanced detector are 
common mode. This enables several configurations in which only one modulator and one transmission fiber are required, as discussed later.

\section{OpticAl FM AND FREQUENCY Discriminators}

We have achieved a close approximation to the ideal transfer functions using custom-fabricated FBGs, as described in Section IV. An optical FM signal centered on the zero- $R$ threshold for each FBG is converted to approximately halfwave-rectified IM with high even-order distortion. However, these even-order distortions cancel (ideally) at the output of the balanced detector.

Selection of the optical frequency range (or ramp bandwidth between OFF and ON) of the optical discriminators is based on several factors. Typically, the link must operate over a range of input RF power levels. For low levels, the resulting modulated optical spectrum (small modulation index) is dominated by the carrier, with some power in the first-order sidebands separated by the modulation frequency from the carrier. For this condition, one would ideally attempt to minimize the ramp bandwidth to maximize the conversion of the small-index FM to IM. For large input signals, the resulting large-FM-index optical spectrum has a significant power in multiple sidebands, extending to several times the modulation frequency. In this case, the ramp bandwidth must be larger to avoid distortion and clipping. Our selection of $10 \mathrm{GHz}$ was a first attempt, seeming a reasonable compromise for operation at modulation frequencies near $2 \mathrm{GHz}$.

For a single-frequency source at frequency $\omega$, phase modulated (PM) with a sinusoidal voltage with a peak voltage $\nu_{o}$ at $\mathrm{RF}$ frequency $\Omega$, the optical field can be expressed as

$$
E(t)=E_{o} \cos \left(\omega_{o} t+\frac{\pi \nu_{o}}{\nu_{\pi}} \cos \Omega t\right)
$$

The instantaneous frequency is the derivative of the phase

$$
\omega(t)=\omega_{o}-\frac{\pi \Omega \nu_{o}}{\nu_{\pi}} \sin \Omega t
$$

which has a peak frequency deviation

$$
\Delta \omega=\frac{\pi \Omega \nu_{o}}{\nu_{\pi}} .
$$

Hence, for narrowband signals, PM results in an equivalent FM with a frequency deviation that is proportional to the modulation frequency. The FM index $\beta=\Delta \omega / \Omega=\pi \nu_{o} / \nu_{\pi}$ is then independent of the modulation frequency. For narrowband RF signals, the frequency dependence of the FM index is of little concern. For wideband signals, this results in a frequencydependent response. To compensate for this, an integrator could be used before the modulator. This integration would then be differentiated by PM, resulting in a flat FM response. Alternatively, an equalizer could be used after detection.

A simple time-domain view of operation of this link provides a reasonable qualitative understanding. The instantaneous optical frequency is linearly proportional to the instantaneous voltage applied to the PM. Each of the complementary frequency discriminators rejects all optical power on one side of the carrier and the carrier itself. Power on the other side is reflected with a strength that is linearly proportional to the instantaneous voltage. Hence, the resulting input-output transfer function is ideal for class B. This simple view is accurate for large $\beta$, for which the optical carrier is swept through a large frequency deviation at a low modulation frequency.

A rigorous analysis [12] of the interaction between the FM signal and the ideal discriminator transfer functions reveals quantitative understanding. For a single modulating sinusoidal signal, the field reflected from each discriminator is described by multiplying the input FM field (1), which is expressed as the well-known series of Bessel functions

$$
E(t)=E_{o} \sum_{n=-\infty}^{\infty} J_{n}(\beta) \cos \left(\omega_{o} t+n \Omega t\right)
$$

by the ideal discriminator responses for the two complementary filters 1 and 2

$$
\begin{aligned}
& H_{1}(\omega)=0, \quad \omega<\omega_{o} \\
& H_{1}(\omega)=\frac{1}{\sqrt{n_{o} \Omega}} \sqrt{\left(\omega-\omega_{o}\right)} e^{j \omega \tau}, \quad \omega_{o}<\omega<\omega_{o}+n_{o} \Omega \\
& H_{1}(\omega)=e^{j \omega \tau}, \quad \omega>\omega_{o}+n_{o} \Omega \\
& H_{2}(\omega)=0, \quad \omega>\omega_{o} \\
& H_{2}(\omega)=\frac{1}{\sqrt{n_{o} \Omega}} \sqrt{\left(\omega_{o}-\omega\right)} e^{j \omega \tau}, \quad \omega_{o}>\omega>\omega_{o}-n_{o} \Omega \\
& H_{2}(\omega)=e^{j \omega \tau}, \quad \omega<\omega_{o}-n_{o} \Omega .
\end{aligned}
$$

The slope of the frequency discriminator is defined by $n_{o}$, such that the filter loss is one when $\omega=\omega_{o} \pm n_{o} \Omega$. We select $n_{o}$ such that negligible power exists in sidebands beyond $n_{o} \Omega$. We assume a constant group delay $\tau$. Clearly, the filter sharp discontinuities shown in Fig. 2 cannot have a perfectly constant group delay, particularly in the vicinity of the discontinuities. However, as discussed in Section IV, our filters have group delay variations of less that approximately $10 \mathrm{ps}$ over the frequencies of interest. This appears to be sufficient in justifying the assumption of a constant $\tau$.

Square-law detection of the filtered spectra provides the photocurrent generated in each detector. A key result from [12] is that the ideal linear power reflectivity-versus-frequency curve does not result in an ideal half-wave rectification, as suggested by the simple time-domain view. Rather, in addition to the signal component, the output includes a dc component as well as a nonlinear distortion. This dc component is in addition to the minimum level of $\mathrm{dc}$ considered for an ideal class B [11]. Fig. 3 shows the signal power, dc, and second- and thirdorder distortion for a single sinusoidal modulation signal as a function of $\beta$ at the output of one of the detectors for $n_{o}=5$. At the output of the balanced detector, the signal and thirdorder distortion from each detector will add, while second-order distortion and dc cancel. However, noise associated with the dc generated in each detector will add, leading to a noise penalty relative to a perfect class $B$. 


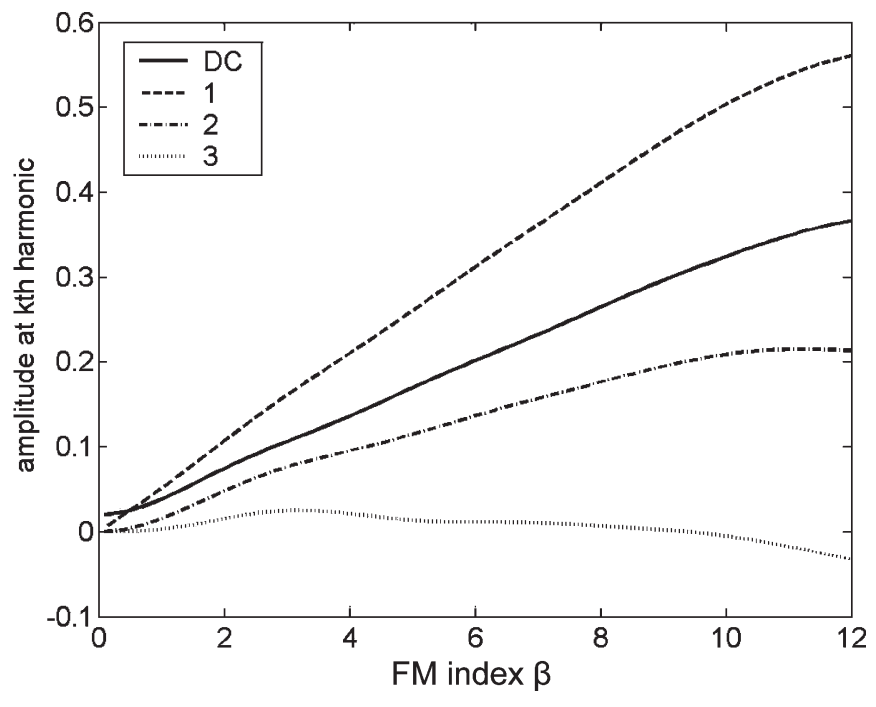

Fig. 3. Predicted response [12] of an ideal FBG discriminator with a carrier suppression of $14 \mathrm{~dB}$ as a function of the FM modulation index and for a modulation with a single sinusoid.

To understand the behavior described by Fig. 3, consider the cases of large and small FM index $\beta$. For a small $\beta$, both the $\mathrm{dc}$ and resulting signal are small. The modulated optical spectrum is dominated by the carrier, with the small sidebands separated from the carrier by the modulation frequency $\Omega$ and smaller sidebands separated by $2 \Omega$. After each filter, the carrier is rejected, leaving sidebands at $\Omega$ and $2 \Omega$, with each attenuated by the square root of the frequency from the carrier. This mix in one of the photodetectors to generate the RF signal at frequency $\Omega$. Since the magnitudes of the first and second sidebands are proportional to $\beta$ and $\beta^{3}$, respectively, the RF output current is proportional to $\beta^{3}$ for small $\beta$.

Leakage of the carrier through the filter introduces an important exception to this scaling. In this case, the residual carrier, which is constant for a small $\beta$, also mixes with the first sideband, creating a signal current at $\Omega$ that is linearly proportional to $\beta$. For a small $\beta$, this leakage can easily dominate the $\beta^{3}$ dependence. Fig. 3 includes the effect of this small carrier leakage, as can be seen by the nonzero dc power at $\beta=0$ and the linear dependence on $\beta$. The carrier leakage here corresponds to a shift of the ideal bias point of 0.2 times the modulation frequency. For $n_{o}=5$, this corresponds to a carrier suppressed by $96 \%$ or $14 \mathrm{~dB}$, rather than the ideal $100 \%$.

Mixing between pairs of sidebands also creates distortion. In general, the signal and each order of distortion result from the superposition of mixing between multiple pairs of sidebands in the Bessel function expansion. For a small $\beta$, mixing between the first and third sidebands creates a second-order distortion at an RF frequency of $2 \Omega$. Mixing between the first and fourth sidebands creates a third-order distortion at $3 \Omega$. Again, carrier leakage, particularly for a small $\beta$, results in additional mixing components. For example, the carrier can mix with the second sideband to create second-order distortion at $2 \Omega$. Even-order distortion cancels after the balanced detector, but odd-order distortion remains.

For a large $\beta$, the dc component and signal increase linearly with $\beta$, with a ratio of signal $/ \mathrm{dc}=\pi / 2$, as expected from a half-

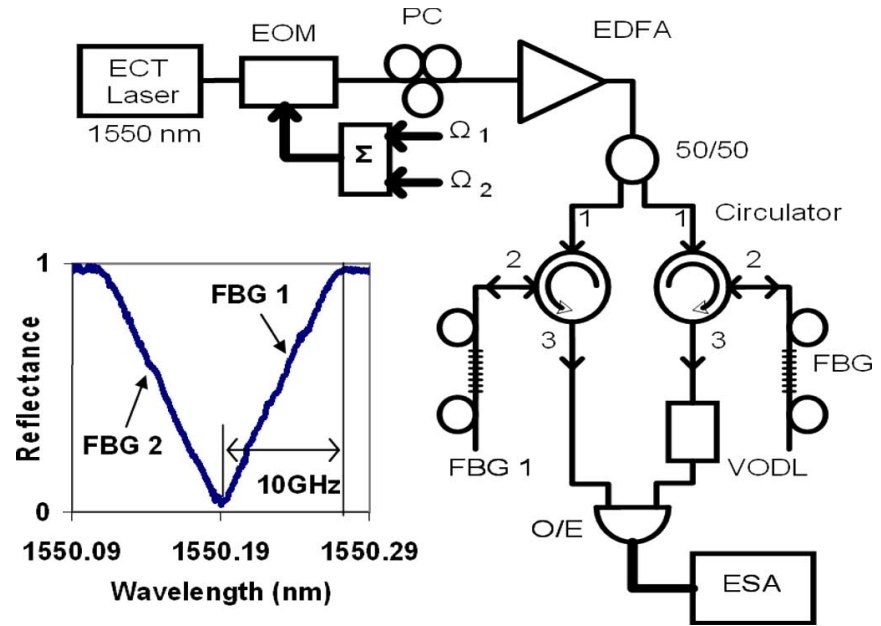

Fig. 4. Experiment setup showing fiber gratings (FBG1 and FBG2), phase modulator (EOM), VODL, and balanced differential detector (O/E). PC is a fiber polarization controller. Inset shows the total reflectivity for the two gratings.

wave-rectified sine wave. The even-order distortion is large, but the odd-order distortion becomes small. This corresponds to an FM modulation with a high frequency deviation but low modulation frequency, as suggested by the time-domain view of the system operation. Operation in this range of $\beta$ is desirable both for noise and linearity and would approximate the ideal class-B link [11]. However, practical constraints, including optical channel bandwidth and modulator drive voltage, preclude operation with $\beta>2$ to 3 .

For a large $\beta$, a significant power exists in the high-order sidebands that may extend beyond the linear range of the grating. For Fig. 3, with $n_{o}=5, \beta>8$ results in a substantial clipping of all components and an additional nonlinear distortion.

For the intermediate values of FM index $\beta$, the situation is complex. The magnitude of each component (Fig. 3) is the result of the superposition of many terms in the squarelaw-detected filtered Bessel-function representation. Both the additional $\mathrm{dc}$ and third-order distortion become problematic. Analysis must also include carrier leakage, as the presence of a small residual carrier introduces additional mixing components that affect all components. We describe in Section V how a third-order distortion is managed and what the impact of dc is on noise.

\section{EXPERIMENT}

Our experimental configuration is shown in Fig. 4. Light from a tunable laser is phase modulated using a 10-GHz lithium niobate modulator ( $V \pi=6 \mathrm{~V}$ and loss $\sim 3 \mathrm{~dB}$ ) with the combined outputs from two signal generators and then amplified using an erbium-doped fiber amplifier (EDFA) with a maximum output power of $17 \mathrm{dBm}$. For the narrowband RF signals near the frequency $\Omega$, PM and FM are related easily using a frequency deviation that is proportional to $\Omega$, as discussed in Section III. Rather than equalizing to compensate for this frequency dependence of the frequency deviation, we operate with an FM index that is independent of frequency and accept 


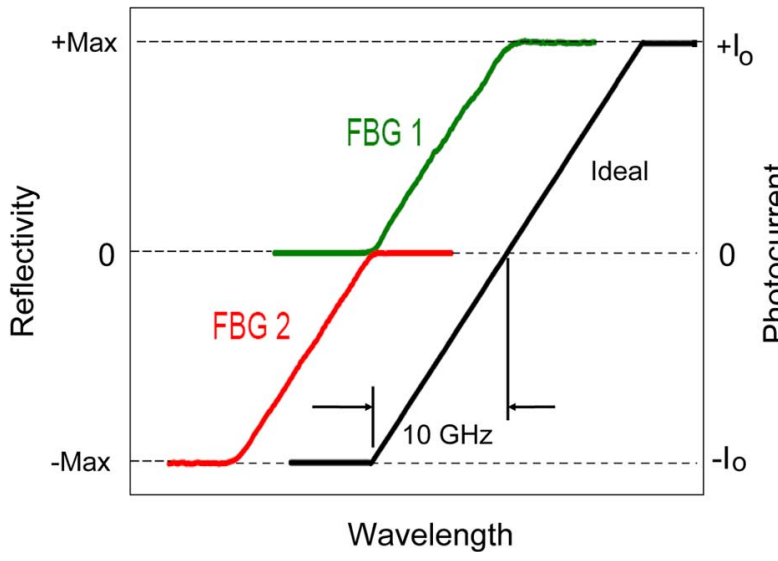

Fig. 5. Measured reflectivity versus optical frequency for FBGs FBG1 and FBG2, with FBG2 inverted, compared to an ideal straight line.

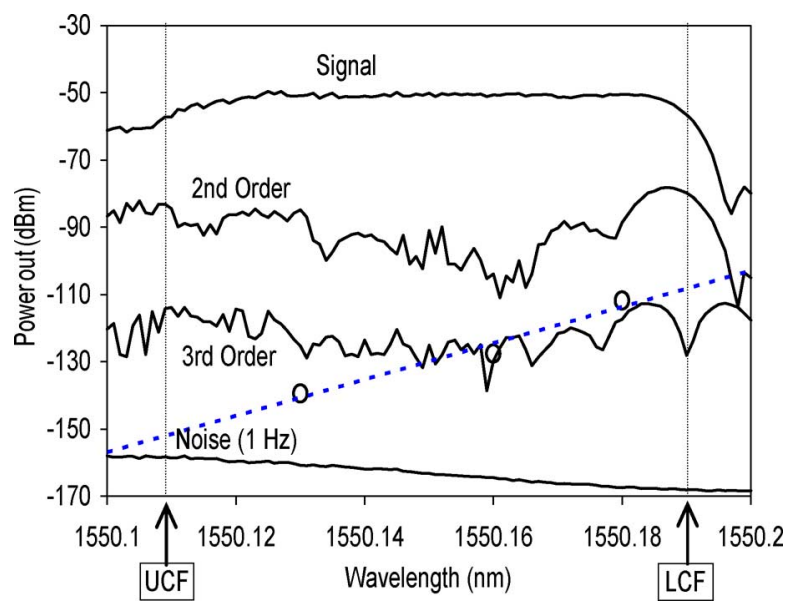

Fig. 6. Frequency response of the RF powers of the signal, second-order distortion, third-order distortion, and noise (1-Hz bandwidth). Two RF tones are at $1.061\left(\Omega_{1}\right)$ and $1.081 \mathrm{GHz}\left(\Omega_{2}\right)$. The signal power is measured at $\Omega_{1}$. The second-order distortion is measured at $2.142 \mathrm{GHz}\left(\Omega_{1}+\Omega_{2}\right)$. The thirdorder distortion is measured at $1.041 \mathrm{GHz}\left(2 \Omega_{1}-\Omega_{2}\right)$.

the small frequency dependence across our narrow operating frequency range.

The FBGs were fabricated by Redfern Optical Components, targeting constant group delay and a linear power reflectivity $R$ over a $10-\mathrm{GHz}$ frequency ramp. Maximum $R$ of each $13-\mathrm{cm}-$ long grating is $94 \%$. Each FBG serves as a good approximation to an ideal transfer function, as shown in Fig. 5. When properly aligned and differenced (class B), the two gratings provide a linear and well-matched discriminator pair. Group delay variations are typically \pm 10 ps over the linear portions of the response curve, with larger variations at the corners and over the portions with flat frequency response.

When measured with a single FM-modulated source tuned across the linear ramp [13], as shown in Fig. 6, a single grating shows an even-order distortion that rises near the threshold at zero $R$ [lower corner frequency (LCF) on the figure]. Thirdorder (two-tone) distortion has a null at frequency corresponding roughly to where the IM response of the fundamental is reduced by $6 \mathrm{~dB}$. This point corresponds to the optimum bias point for each grating. Also, evident in Fig. 6 is the decrease

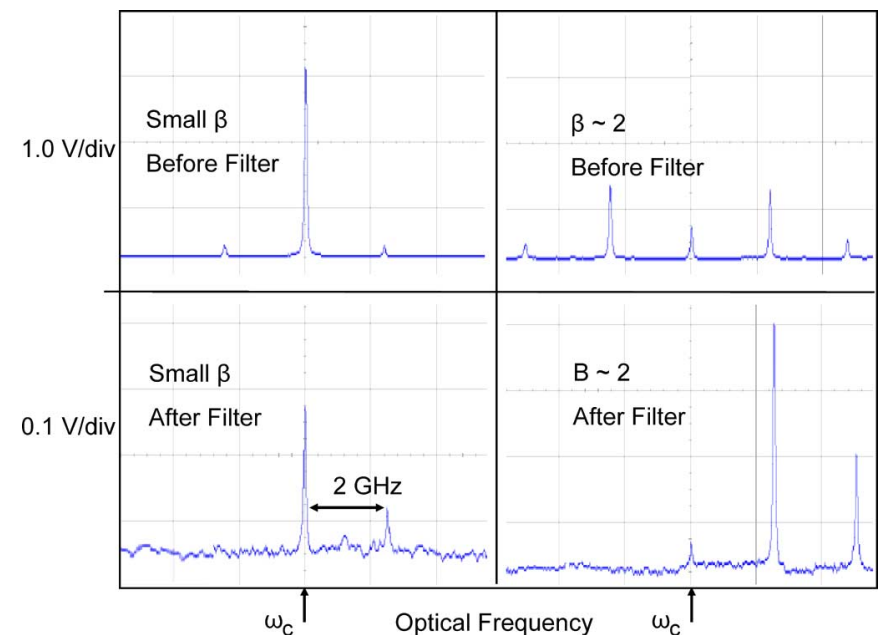

Fig. 7. Optical spectra measured using scanning Fabry-Pérot etalon before (upper) and after (lower) Bragg-grating filter (FBG2), for low (left, $\beta \sim 0.1$ ) and high (right, $\beta \sim 2.0$ ) FM modulation index.

in carrier-related noise that accompanies tuning the FM source from the upper corner frequency to the LCF. The dotted line shows the third-order distortion for an MZ operated at the same modulation depth.

The two FBGs are temperature or strain tuned to alignment, as shown in Fig. 5. Ultimately, the gratings would be stabilized with active control, but for demonstration purposes, tuning was done manually using a micrometer to introduce strain. Reflected signals are separated from the input signals using circulators, matched in RF phase using a variable optical delay line (VODL), and detected in a 20-GHz balanced photodetector (Discovery Semiconductors). Computer control of signal generators and the RF spectrum analyzer enabled rapid measurement of signal, linearity, and noise, minimizing problems with frequency drift of the gratings.

Fig. 7 shows the optical spectrum as measured on a confocal scanning Fabry-Pérot etalon before and after the filter FBG2 biased at the optimum bias point for a third-order distortion. For a small $\beta$, the carrier is the dominant feature with little power in modulation sidebands. While this is clearly an inefficient mode of operation (in terms of power), a similar inefficiency occurs in conventional subcarrier modulation in which IM depths of a few percent are common. For a large $\beta$, the power in the sidebands is comparable to that of the carrier. For $\beta=2.4$, the carrier is suppressed to zero. At the filter bias point corresponding to the minimum in a third-order distortion, the carrier is suppressed by approximately $11.3 \mathrm{~dB}$.

\section{Results AND Discussion}

Fig. 8 shows the total measured noise and noise corrected for analyzer and receiver noise. The combination of shot and intensity noises increases with an increasing RF power, as ideally, the only power transmitted is the signal power. For a Q-biased MZ, this noise should be constant. However, our grating transfer functions do not have perfectly sharp thresholds, and optimum results (minimum third-order distortion) are 


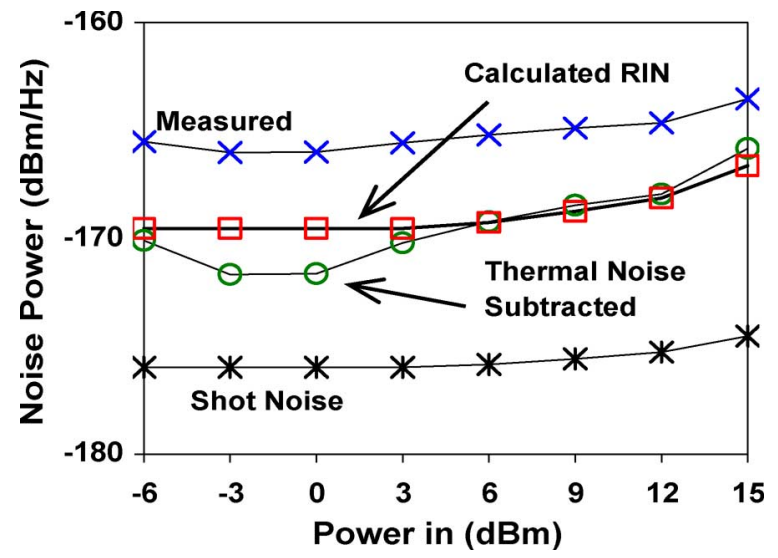

Fig. 8. Measured RF noise power $(\mathbf{X})$ as a function of the RF power. Calculated shot noise $(*)$ and RIN $(\square)$ are based on the total measured photocurrent and an RIN of $-146 \mathrm{~dB} / \mathrm{Hz}$. Correcting the measured noise for the analyzer and receiver noise results in (o).

obtained with gratings tuned such that a total photocurrent of $630 \mu \mathrm{A}$ is received with a zero RF applied.

Based on the discussion of Section III, the carrier admitted by the filter at the optimum operating point has a significant effect on the link performance (for a low $\beta$ ). First, the resulting RF photocurrent is dominated by the beating between the carrier and first-order sidebands; hence, it is proportional to $\beta$ rather than $\beta^{3}$, as predicted ideally. This one-to-one scaling between the input and output RF link power is what would be expected for conventional links. Second, the third-order distortion generated by the beating between the carrier and third-order sidebands scales as $\beta^{3}$, whereas a higher-dependence would result $\left(\beta^{5}\right)$ for the perfect filters.

In general, the third-order distortion resulting from the FM-to-IM conversion (Fig. 3) and that resulting from the imperfect linearity of the FBG filters combine. Fortunately, adjusting the bias point of each FBG in the vicinity of the turn-on can result in a wide range in magnitude and sign of the third-order nonlinearity, such that a point can generally be found at which these two effects cancel, as shown in Fig. 6 by the point "LCF."

Fig. 9 shows the measured RF performance. An SFDR of $110.43 \mathrm{~dB} \cdot \mathrm{Hz}^{2 / 3}$ was achieved (third-order, based on total noise corrected for analyzer and receiver noise). Given the relative intensity noise (RIN) of our laser and EDFA combination $(-146.5 \mathrm{~dB} / \mathrm{Hz})$, the maximum SFDR that could be achieved using a conventional Q-biased MZ would be approximately $100 \mathrm{~dB} \cdot \mathrm{Hz}^{2 / 3}$. Hence, our class-B link shows far greater tolerance to RIN. Also, this SFDR was achieved for a total received photocurrent of only $880 \mu \mathrm{A}$. A Q-biased-MZ link operating with a source laser RIN of $-160 \mathrm{~dB} / \mathrm{Hz}$ would require $10 \mathrm{~mA}$ of received photocurrent to achieve the same SFDR. This demonstrates clearly the ability of class B to suppress carrierrelated noise and improve link performance dramatically.

Results presented in Fig. 9 are for transmission over a few meters of fiber only. Measurements were also conducted over 10 and $20 \mathrm{~km}$ of standard single-mode fiber. Since the carrier is suppressed substantially by the FBGs prior to detection, the impact of signal-spontaneous beat noise is reduced substantially. Measurements were taken with the EDFA at the output

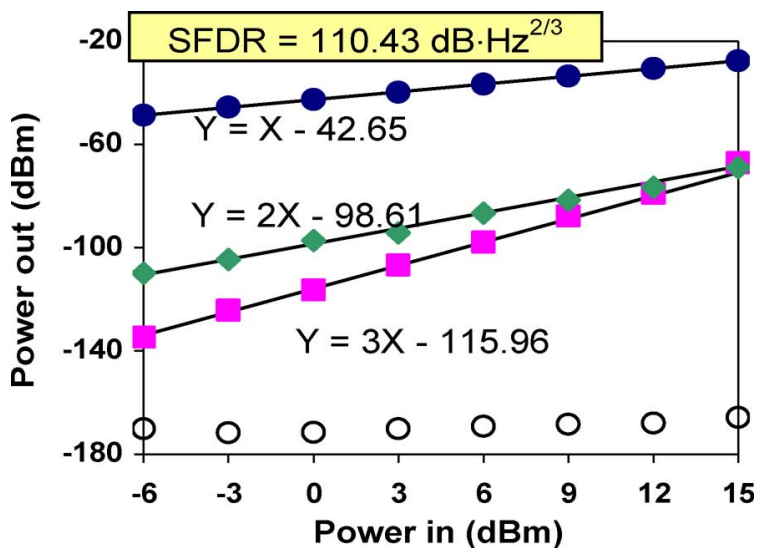

Fig. 9. RF power out versus RF power in (signal generator output) for class-B measurement showing the signal $(\bullet)$, second-order sum frequency $(\bullet)$, thirdorder two-tone ( $\square$ ), and noise corrected for the receiver and analyzer (o). RF modulation frequencies are 2091 and $2111 \mathrm{GHz}$.

of the transmission fiber, which is a configuration that would result in intolerable noise in a conventional class-A system since the effective noise figure increases as the power at the EDFA input decreases. Results were nearly identical to Fig. 9, with SFDR values between 110 and $111 \mathrm{~dB} \cdot \mathrm{Hz}^{2 / 3}$. Variability was dominated by a drift of our manually controlled gratings.

Notably, since the conventional approach to intensity noise suppression using dual-output MZs results in uncorrelated noise at the two detectors, it is not possible to amplify after the modulator. With our class-B FM approach, we not only use just one modulator and transmission fiber but also are able to use optical amplifiers anywhere in the span while retaining a low intensity noise.

Our grating was designed with a very high frequency selectivity (10 GHz off-to-on) for an operation near $2 \mathrm{GHz}$. Increasing the operating frequency or FM index would suggest gratings with a lower frequency selectivity. Since frequency selectivity translates into grating length, fabricating linear gratings with 30-50-GHz slopes and sharp turn-on would be less challenging than our $10-\mathrm{GHz}$ gratings. Therefore, we expect operation at higher frequencies (e.g., $\mathrm{X}$-band) to result in a better grating performance. However, extending operation to $\mathrm{X}$-band or higher will create challenges with fiber dispersion (chromatic or polarization mode), particularly for a large FM index where the modulated source spectrum is broad.

Several other considerations with class-B operation should be noted. The FBGs can be located in the transmitter, with two matched transmission fibers, or the FM signal can be transmitted on one fiber to gratings located at the receiver. In principle, even-order distortion generated in the FM-to-IM conversion cancels in balanced detection. This would allow broadband operation for applications in which even-order distortion products are in band. However, achieving a high cancellation over broad frequency ranges will be challenging. Second-order distortion generated in each branch must cancel to an appropriate level over the desired broad frequency range. Devices like push-pull amplifiers and electronic predistortion circuits accomplish this balance routinely. Optical feedforward linearization techniques have also been made to work successfully. Feedforward or 
electronic predistortion does not, however, benefit from the noise reduction associated with carrier suppression. We reserve judgement as to the ability of our approach to meet strict broadband requirements.

Balancing power levels and phase of the two paths adds complexity, but this must be done also in alternative approaches for achieving a high SFDR (dual-output MZ).

Crossover distortion is a challenge for electronic class- $\mathrm{B}$ systems, but was not apparent or problematic in our system. Finally, we expect a small residual wavelength dependence of the FBG group delay to result in distortion at higher frequencies. None of this was apparent at our operating frequencies near $2 \mathrm{GHz}$. Work continues to understand the ultimate design objectives and performance in the presence of these effects.

\section{CONCLUSION}

We present the first demonstration of a class-B MPL, achieving an SFDR of $110.4 \mathrm{~dB} \cdot \mathrm{Hz}^{2 / 3}$ with less that $0 \mathrm{dBm}$ received optical power and showing a dramatically increased tolerance of the intensity noise. Two-tone tests were conducted at $2.1 \mathrm{GHz}$, with a frequency separation of $20 \mathrm{MHz}$. Nearly ideal transfer functions for complementary modulated sources were realized using optical FM and special FBGs. By suppressing the optical carrier (11.3 dB demonstrated) while retaining a high modulation efficiency, class B provides linear and lownoise transmission in which, in strong contrast to conventional links, most of the received optical power is signal power (for a large FM index). Notably, this allows reduction of intensity noise without having to resort to two transmission fibers and is effective in suppressing intensity noise that is uncorrelated in the balanced detectors (e.g., from optical amplifiers).

Ideal class $\mathrm{B}$ represents the theoretical maximum performance for an RF link. While our results are encouraging, a considerable work remains to understand the ultimate performance and limitations of the class-B FM approach. A continued refinement in the system and optical frequency discriminator design should provide links with unprecedented dynamic range.

\section{REFERENCES}

[1] C. H. Cox, III, Analog Optical Links: Theory and Practice. Cambridge, U.K.: Cambridge Univ. Press, 2004.

[2] L. T. Nichols, K. J. Williams, and R. D. Esman, "Optimizing the ultrawide-band photonic link," IEEE Trans. Microw. Theory Tech., vol. 45, no. 8, pp. 1384-1389, Aug. 1997.

[3] R. D. Esman and K. J. Williams, "Wideband efficiency improvement of fiber optic systems by carrier subtraction," IEEE Photon. Technol. Lett., vol. 7, no. 2, pp. 218-220, Feb. 1995.

[4] A. C. Lindsay, "An analysis of coherent carrier suppression for photonic microwave links," IEEE Trans. Microw. Theory Tech., vol. 47, no. 7, pp. 1194-1200, Jul. 1999.

[5] T. E. Darcie and P. P. Ianonne, "Method and apparatus for laser performance enhancement," U.S. Patent 6181 453, Jan. 30, 2001.

[6] S. Mathai, F. Cappelluti, T. Jung, D. Novak, R. B. Waterhouse, D. Sivco, A. Y. Cho, G. Ghione, and M. C. Wu, "Experimental demonstration of a balanced electroabsorption modulated microwave photonic link," IEEE Trans. Microw. Theory Tech., vol. 49, no. 10, pp. 1956-1961, Oct. 2001.

[7] T. E. Darcie and A. Moye, "Modulation-dependent limits to intensitynoise suppression in microwave-photonic links," IEEE Photon. Technol. Lett., vol. 17, no. 10, pp. 2185-2187, Oct. 2005.

[8] W. K. Burns, G. K. Gopalakrishnan, and R. P. Moeller, "Multi-octave operation of low-biased modulators by balanced detection," IEEE Photon. Technol. Lett., vol. 8, no. 1, pp. 130-132, Jan. 1996.
[9] T. E. Darcie, A. Moye, P. F. Driessen, J. Bull, H. Kato, and N. A. F. Jaeger, "Noise reduction in class-AB microwave-photonic links," in Proc. IEEE Int. Top. Meeting MWP, Seoul, Korea, Oct. 12-14, 2005, pp. 329-332.

[10] J. D. Bull, T. E. Darcie, J. Zhang, H. Kato, and N. A. F. Jaeger, "Broadband class- $\mathrm{AB}$ microwave-photonic link using polarization modulation," IEEE Photon. Technol. Lett., vol. 18, no. 9, pp. 1073-1075, May 2006.

[11] T. E. Darcie and P. F. Driessen, "Class-AB techniques for high-dynamicrange microwave-photonic links," IEEE Photon. Technol. Lett., vol. 18, no. 8, pp. 929-931, Apr. 15, 2006.

[12] P. F. Driessen, T. E. Darcie, and J. Zhang, "Analysis of class-B microwavephotonic link using optical frequency modulation,” Jan. 2007. submitted for publication.

[13] J. Zhang and T. E. Darcie, "Low-biased microwave-photonic link using optical frequency or phase modulation and fiber-Bragg-grating discriminator," presented at the Optical Fiber Commun./National Fiber Optic Engineers Conf. (OFC/NFOEC), Anaheim, CA, Mar. 6-10, 2006 , Paper OWG1.

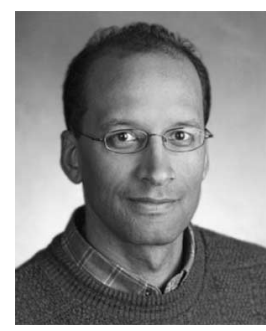

Thomas E. Darcie (F'00) received the B.Sc. degree in physics from the University of Waterloo, Waterloo, ON, Canada, in 1978 and the M.Sc. and $\mathrm{Ph} . \mathrm{D}$. degrees in aerospace from the University of Toronto, Toronto, ON, in 1982.

He joined the technical staff of AT\&T Bell Laboratories, Holmdel, NJ, to study a wide variety of topics related to lightwave telecommunications, including fiber fabrication processes, semiconductor lasers, optical amplifiers, and numerous modulation and multiplexing techniques. He has been a lead figure in the development of lightwave systems for analog applications in cable television and wireless systems. As the Head of access communications research at AT\&T Bell Laboratories from 1989 to 1995, he was responsible for technology innovation in wireless, lightwave, and hybrid fiber-coax systems. As Vice President of AT\&T Laboratories and Director of the Communications Infrastructure Research Laboratory from 1995 to 2002, he led a research laboratory that provided technology support for AT\&T's diverse requirements in optical networking, broadband access, fixed wireless access, wireless local-area networks, and cellular systems. He is currently a Professor at the University of Victoria, Victoria, BC, Canada, and holds a Tier 1 Canada Research Chair. $\mathrm{He}$ has more than 100 technical publications and 30 patents spanning the aforementioned broad set of technologies. His research program focuses on systems for communications, imaging, and sensor applications.

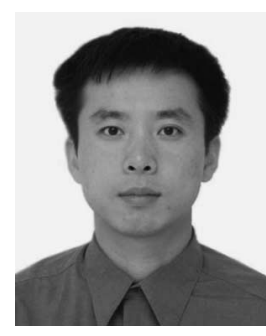

Jinye Zhang received the B.Sc. and M.Sc. degrees in physico-electronics from Tianjin University, Tianjin, China, in 1994 and 2000, respectively, and the $\mathrm{Ph} . \mathrm{D}$. degree in electrical engineering from the Northwestern University, Evanston, IL, in 2005.

Since 2005, he has been a Postdoctoral Fellow at the Optical Systems and Technology Laboratory, University of Victoria, Victoria, BC, Canada. His research interests include nonlinearities in optical fibers and fiber-optic systems for communications and sensing.

Dr. Zhang is a member of the Optical Society of America. 


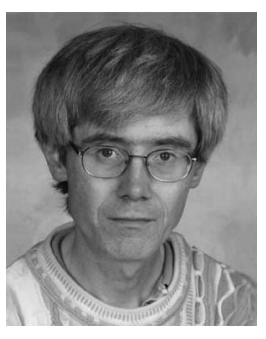

Peter F. Driessen (SM'93) received the B.Sc. degree in physics and the Ph.D. degree in electrical engineering from the University of British Columbia, Vancouver, BC, Canada, in 1976 and 1981, respectively.

$\mathrm{He}$ was with various companies in Vancouver designing modems for five years before joining the University of Victoria, Victoria, BC, where he is currently a Professor at the Department of Electrical and Computer Engineering and cross-appointed to the Department of Computer Science and the School of Music. He spent two sabbaticals and several summers at AT\&T Bell Laboratories, Holmdel, NJ, working on various aspects of wireless communications systems. He has more than 80 technical publications and nine patents. His research interests include audio and video signal processing, computer music, sound recording, wireless communications, and radio propagation.

Dr. Driessen was an Editor for the IEEE TRANSACTIONS ON WIRELESS COMMUNICATIONS from 1999 to 2004.

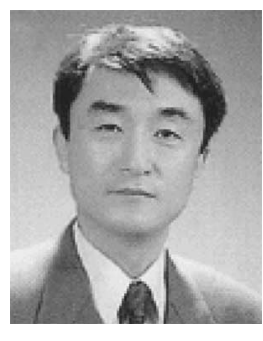

Jae-Jeong Eun received the B.S., M.S., and Ph.D. degrees from Yonsei University, Seoul, Korea.

Since March 1989, he has been with the Department of Electronic Engineering, Changwon National University, Changwon, Korea, as a Professor. He is currently a Research Associate at the Department of Electrical and Computer Engineering, University of Victoria, Victoria, BC, Canada. His recent research interests include microwave photonic links, characterization and application of fiber Bragg gratings, and optical fiber sensors. 